\title{
Kernel-Based Traffic Sign Tracking to Improve Highway Workzone Recognition for Reliable Autonomous Driving
}

\author{
JongHo Lee*, Young-Woo Seo, Wende Zhang, and David Wettergreen
}

\begin{abstract}
To be deployed in the real world, a self-driving car must be capable of responding to exceptional road conditions, such as temporary work zones, because such events can change previously known traffic rules and road geometry. To develop such a capability, we implemented a computer vision system that recognizes the bounds of highway workzones by detecting regulatory and warning workzone signs. Because it is not practical to expect perfect performance in sign recognition, we also developed a confidence-propagation method to handle potential sign recognition errors. The performance of highway workzone recognition was improved by confidence-propagation, but our approach is not easily scalable to some real-world scenarios. Instead of only propagating sign classification confidence, in this work we project the appearance information of previously detected signs onto the current frame, to constrain the region for searching. Through experiments, we show that kernel-based tracking reduced the miss and false detection rates, result in a better performance of highway workzone recognition. In this paper, we present our on-going effort to further improve the performance of our highway workzone recognition system.
\end{abstract}

\section{INTRODUCTION}

For the past several decades, research efforts in the development of self-driving cars have drastically improved related technologies. For example, researchers have advanced consumer cars from early approaches such as equipping them with $\mathrm{B} \& \mathrm{~W}$ cameras, which merely follow road-markings at a very low speed, to developing a semi-autonomous vehicle that can drive on highway routes stretching thousands of kilometers and containing normal traffic [6], [18] and to designing fully autonomously vehicles that drive on 60 milelong (mock-) city routes while interacting with other robotic vehicles [15], [19]. In the near future, these technological advancements will help us realize self-driving cars that can function in daily life.

Before fully-autonomous vehicles can be available for daily use, they must at least be able to cope with the most plausible driving environment for self-driving car operations: highways where road geometry is less variable, where traffic rules are clearly visible and where direct interactions with other vehicles are infrequent. The benefits of deploying selfdriving cars on highway are also quite tangible as well in that operating a self driving car is expected to reduce a large

\footnotetext{
*JongHo Lee is with Mechanical Engineering, Carnegie Mellon University, 5000 Forbes Ave., Pittsburgh, PA 15213, USA. jonghole at andrew. cmu . edu

Young-Woo Seo and David Wettergreen are with the Robotics Institute, Carnegie Mellon University, 5000 Forbes Ave., Pittsburgh, PA 15213, USA $\{$ ywseo,dsw $\}$ at ri.cmu.edu

Wende Zhang is with the Electrical and Controls Integration Lab, General Motors R\&D, 30500 Mound Rd, Warren, MI 48092, USA. wende.zhang at gm.com
}

number of highway traffic accidents caused by lack of human drivers attention and inefficient gas consumption. In addition, self-driving vehicles would facilitate more efficient public freight transportation. Finally, for automated transportation, deployment of self-driving cars on highways would be less expensive than building new infrastructure on highway.

On a highway, the driving behavior of an autonomous vehicle is primarily guided by two properties of a highway: its road geometry and the traffic rules. A map of the highway can be manually or automatically prepared in advance to provide the vehicle with detailed information about road geometry and traffic rules. However, it is not possible to describe unexpected occurrences on a map in a priori, such as traffic accidents or road work. For safe and reliable autonomous driving, the vehicle must be able to effectively handle such events, which may cause temporary changes in road conditions.

To effectively handle unexpected events on a highway, an autonomous vehicle should first be able to identify the bounds, e.g., the beginning/end of a workzone, and understand possible changes an unexpected event imposes on the highway, e.g., decrease in speed or blockage of a lane. We developed a color-based sign detection system to recognize work-zones on highways [16]. In particular, our system learns variations of a color in sign images so as to perform a pixel-wise binary color classification. Then our method identifies blobs to localize sign image regions. Finally, our method represents a sign image in a homogeneous feature space in order to reduce variation of geometric shapes. To handle potential sign recognition errors, we exploit temporal redundancy of sign appearances in such a way that signrecognition confidence is forwarded to smooth out the effect of any signs missed. Because we propagate only the confidence, and not the appearance information about the detected signs, our workzone recognition system can be upgraded by improving our sign detection method.

To improve the performance of our color-based sign detection method, we apply an appearance-based, sign tracking algorithm. In particular, our approach exploits two features of appearance of a sign in a video stream, in which a sign appears multiple times before it disappears from the view point. First, in the two consecutive image frames, there are overlapping regions, particularly in the images of the sign. Second, there are small variations of the appearances and locations. By considering these features, the detected sign and its rectangular region are first given as an input to our tracking system. Then, the detected sign image is modeled with a probability density distribution as a target. 


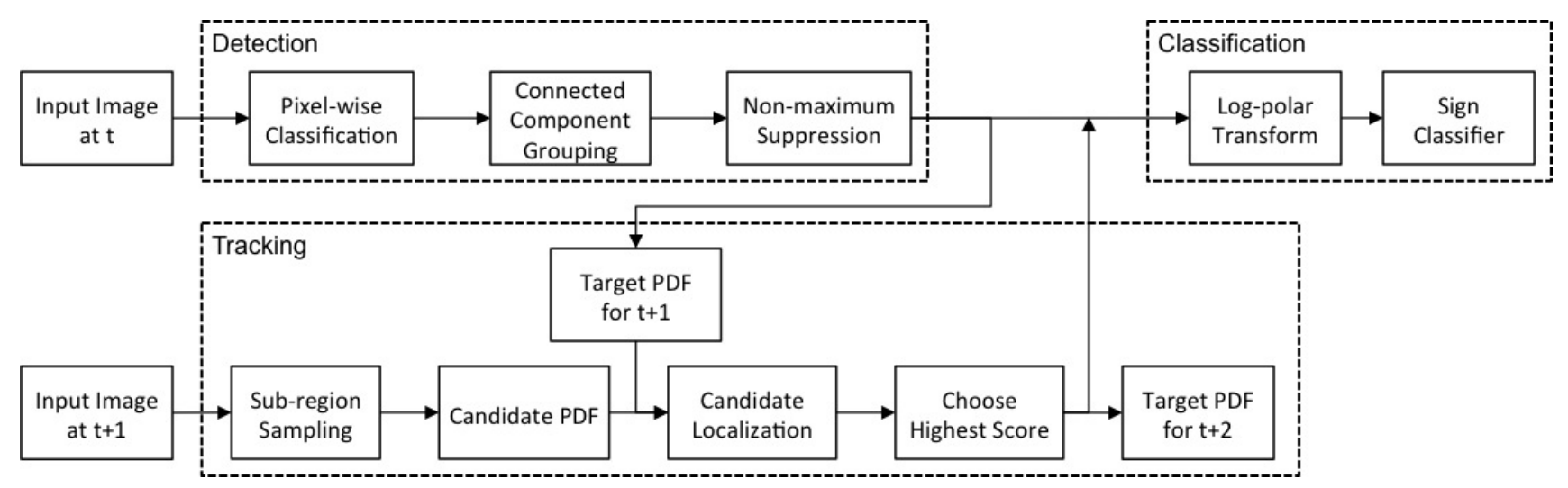

Fig. 1. A schematic overview of our workzone recognition system. Our sign detection module localizes a potential sign in an image at time $t$ and the potential sign is represented as a target probability density for an image at time $t+1$. The image at time $t+1$ and a target probability density are given as inputs to our tracking module. Different sizes of sub-regions of an image at time $t+1$ are represented as candidate probability density and localized by the mean-shift algorithm. A candidate with the highest score to a target is an output as a new target for the subsequent frame. Our classifier estimates the target class of the localized sign from either our detection or tracking.

Next, the rectangular region from the input is projected onto the subsequent frame as a candidate, which is also modeled with a probability density distribution. Lastly, our approach iteratively shifts the rectangular region until the distance between the target and the candidate becomes minimum.

In this paper, we describe a successful application of kernel-based tracking techniques to improve the performance of localizing workzone signs from perspective images and the overall performance of our workzone recognition system.

\section{RELATED WORK}

Many researchers have proposed traffic sign recognition systems. The majority of these systems are composed of two steps: detection and classification.

The color and shape of traffic signs have been used as two dominant cues when searching traffic signs in images. Some of the color-based sign detection methods use colorvalue thresholding to detect the potential signs in an image. For example, several researchers manually investigated color values, while others, including us, used machine learning techniques to obtain the optimal thresholds of target color [13]. The shape-based sign detections usually utilize either the gradient of gray scale image [14] or trained model from data sets [1].

For the classification step, well-known machine learning techniques, such as neural network (NN) [7], linear discriminative analysis (LDA) [1], SURF matching [3], and cross-correlation [17] have been applied. While Eichner and Breckon [7] tried to classify the context of speed limit signs, others tried to classify the appearances of the signs, e.g., color and shape information, rather than their contexts.

Some of the traffic sign recognition systems combine a detection with a tracking system in order to improve the performance. The majority of the tracking systems deal with the dynamics of the tracked signs while the others cope with the appearances of the tracked signs. In order to reduce the search region and improve the detection performance, the discrete-time dynamics of vehicles have been utilized, for example, in Bayesian Filters [8], [11], [13], [14], [17] and information fusion [1], in order to predict the position of a sign in subsequent frames.

On the other hand, the appearance approach for tracking, which is implemented in our approach, is based on the visual appearance [12], in which the well-established Lucas-Kanade Tracker (LKT) [2] has applied. Liu et al. [12] chose several interesting points to overcome the computational cost of LKT and these improvements allowed their algorithm to run in real-time, though there might be problems when images are blurred due to vehicle motion. Instead of requiring complex dynamics of vehicles, our kernel-based object tracking [4] utilizes the appearance from a sequence of images to effectively handle the variations.

\section{RECOGNIZING TEMPORARY TRAFFIC CONTROL SIGNS}

Our task is to understand temporary changes on highways, such as altered road geometry and traffic rules, through workzone sign recognition. The schematic overview of our approach is presented in Fig. 1. Our system is composed of three components including color-based sign detection, kernel-based sign tracking, and sign classification. In the following section, each component of our proposed approach is explained.

\section{A. Sign Detection}

The appearnace of traffic sign is strictly regulated by local and national traffic authorities. For example, all the background color of workzone signs is orange. However it is still challenging to identify an orange colored pixel in an image because aging, wearing, weather, and lighting often cause color variations. To effectively handle these issues, we formulate learning of orange color variation as a binary color classification using the Bayesian inference framework.

$$
P(\operatorname{sign} \mid \mathbf{X})=\eta P(\mathbf{X} \mid \operatorname{sign}) P(\text { sign })
$$

where $\mathbf{X}$ is a total number of $m$-dimensional pixels and $\eta$ is a normalizer for the posterior distribution. We obtain 
the prior probability distribution, $P(\mathrm{sign})$, from the ground truth of our training data, and use AdaBoost [9] to learn the likelihood function, $P(\mathbf{X} \mid$ sign $)$, of a given pixel as part of the target sign. The training data contains 29 images of positive data and 37 images of negative data, some from the web and the rest from our workzone images. A set of weak-classifiers and their weights are trained by these data.

$$
\begin{aligned}
& P(\mathbf{X} \mid \text { sign })=\operatorname{mode}\left(\cup_{j} g\left(f\left(\mathbf{x}_{j} \mid \text { sign }\right)\right)\right) \\
& f\left(\mathbf{x}_{j} \mid \text { sign }\right)=\sum_{i=1}^{H} \alpha_{i} h_{i}\left(\mathbf{x}_{j}\right)
\end{aligned}
$$

where $\mathbf{x}_{j}$ is a 2-dimensional color vector which consists of hue and saturation from pixels in an image, $\mathbf{x}_{j} \in \mathbf{X}, g$ is a function to convert the binary output of AdaBoost into a probabilistic output [10], $g(f(y))=\frac{\exp (f(y))}{\exp (f(y))+\exp (-f(y))}$, $H$ is the number of weak classifiers, $h_{i}$ represents a weak classifier and $\alpha_{i}$ is its weight. We got $97 \%$ pixel-wise detection accuracy using this color classifier.

This color classifier is executed on every pixel to assign a probability for whether the pixel is part of an orange workzone sign or not. Our sign detector runs connectedcomponent grouping algorithm to identify orange blobs and uses non-maximum suppression to select the largest bounding box when the width or height is bigger than 30 pixels. To detect a regulatory, rectangular workzone sign which includes two colors (orange at the top and white at the bottom), we heuristically increase the bounding box based on the aspect ratio.

\section{B. Sign Tracking}

In this section, similar notations to [4] are used with appropriate modifications when necessary. The bounding box of a potential workzone sign from our sign detector is given as an input to our sign tracking component. Since the color variation between two consecutive frames is small, we represent the potential workzone sign, the target, as a probability density distribution by computing a histogram in hue-saturation-value (HSV) color space. We chose HSV color space since it is less affected by illumination when compared to red-green-blue (RGB) color space. In particular, only hue and saturation values are quantized into $n_{h} \times n_{s}$ bins, where $n_{h}$ and $n_{s}$ are the bin numbers of hue and saturation, respectively. To avoid underfitting or overfitting, we choose both $n_{h}$ and $n_{s}$ as 20 . To represent the target model consistently with various sizes of signs, the pixel coordinates should be normalized. Furthermore, to focus on the appearance of a sign rather than the background, we utilize a kernel, $K$, which combines Epanechinikov profile [4] and a mask of the detected sign. In this kernel, the pixel coordinates closer to the center get larger weights and those within mask get weights of $K(\mathbf{p})=c_{k}(1-\mathbf{p}) \cdot$ mask. Fig. 2 illustrates this kernel. Finally, we can calculate the probability density distribution of the target model, $\mathbb{T}=$

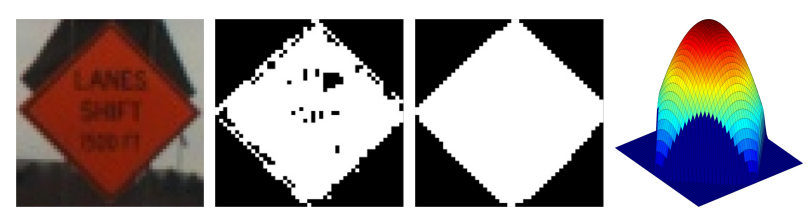

Fig. 2. Kernel functions. The left image is the output of the detected sign, and the middle image is a mask of shape. In order to reduce the background effect and concentrate on the target color, Epanechinikov kernel and the mask of shape from the detection output are combined. The right image illustrates this combined kernel.

$\left\{t_{b}\right\}_{b=1 \cdots n_{h} \times n_{s}}$, on normalized pixel coordinates, $\mathbf{p}$, as

$$
\begin{aligned}
t_{b} & =t_{n_{s} \cdot(j-1)+k} \\
& =C \sum_{i=1}^{n} K\left(\left\|\mathbf{p}_{i}\right\|^{2}\right) H\left(\mathbf{p}_{i}, h_{j}, s_{k}\right)
\end{aligned}
$$

where

$H\left(\mathbf{p}_{i}, h_{j}, s_{k}\right)=\left\{\begin{array}{cc}1 & \text { hue }\left(\mathbf{p}_{i}\right) \in h_{j} \cap \operatorname{sat}\left(\mathbf{p}_{i}\right) \in s_{k} \\ 0 & \text { otherwise }\end{array}\right.$

and $C$ is a normalization constant.

We also need to calculate candidates, $\mathbf{c}(\mathbf{z})$, in the subsequent frame to localize, where $\mathbf{z}$ is the new center. We can calculate candidates same as the target except the new normalized pixel coordinate, $\mathbf{p}_{i}^{\text {new }}$ based on $\mathbf{z}$, because the location of the sign in the subsequent frame shifts. Also, the size of the bounding box increases as the vehicle gets closer to the sign. We can estimate the size of sign in the subsequent frame, but it does not have to be exact. To increase our performance, we apply five different sizes of kernels $(0 \%$, $2 \%, 5 \%, 7 \%$, and $10 \%$ increased kernels) and choose one as the tracking result. $\mathbf{c}(\mathbf{z})$ is calculated by (1) with $\mathbf{p}_{i}^{\text {new }}$.

The distance between these two probability density distributions is the minimum when the candidate model is matched to the target model. Minimizing the distance can be interpreted as maximizing the similarity, and we choose Bhattacharrya coefficient [4], $B(\mathbf{t}, \mathbf{c}(\mathbf{z}))$, to measure the similarity, where $B(\mathbf{t}, \mathbf{c})=\sum_{b=1}^{n_{h} \times n_{s}} \sqrt{t_{b} \cdot c_{b}}$. Using linear approximation around $\mathbf{z}$, we are now able to use the meanshift algorithm [4] to find the mode of

$$
\frac{C_{s}}{2} \sum_{i=1}^{n} w_{i} K\left(\left\|\frac{\mathbf{z}-\mathbf{p}_{i}^{\text {new }}}{\mathbf{s}}\right\|^{2}\right)
$$

where $w_{i}$ is

$$
w_{i}=\sum_{b=1}^{n_{h} \times n_{s}} \sqrt{\frac{t_{b}}{c_{b}(\mathbf{z})}} H\left(\mathbf{p}_{i}^{\text {new }}, h_{j}, v_{k}\right)
$$

We can find the maximum value of (2) by the gradient. If Epanechnikov kernel is used, the gradient of (2) will be a weighted summation as the standard mean-shift algorithm. In our case, though, the mean-shift algorithm is represented as

$$
\mathbf{z}^{\text {new }}=\frac{\sum_{i=1}^{n} \mathbf{p}_{i}^{\text {new }} w_{i} \cdot \text { mask }}{\sum_{i=1}^{n} w_{i} \cdot \text { mask }}
$$

which is still a dot product that has low computational cost. The mean-shift algorithm is repeatedly executed until the 
error is less than the predefined threshold or the number of iterations is less than the maximum iteration number, which is usual. The center position is updated in every iteration by (3). Once all of the candidates converge, we choose the final output which has the highest Bhattacharrya coefficient and provide it to our sign classification component.

\section{Sign Classification}

A bounding box from both our sign detection and tracking as a potential workzone sign is given as an input to our sign classification component. The goal of this research is to recognize temporary changes on workzones and we need to recognize the signs by classifying them. In this purpose, nine different workzone signs are chosen as target classes which indicate a workzone bounds and temporary changes and the remaining workzone signs are assigned to another class. Table I explains the target classes in detail.

Three major issues exist to classify the detected signs. First, the intensities of the detected signs can vary due to the environment. Second, the dimensions of the detected signs are various. The dimension of a sign is increasing when the sign is closer to the camera. Third, it is almost impossible to see the canonical shape of a workzone sign because of the perspective projection into an image. Also, signs can be slightly tilted or rotated while installing. In order to effectively handle these issues, our sign classifier first normalizes the intensity of the detected sign, and then transforms it into a log-polar image. This log-polar transform samples densely image intensity values near the center of a sign image where the distortions are relatively small, and then sparsely collects values from sign image boundaries where the geometric distortions are large. Also, this log-polar transformed image always have $\rho$ by $\theta$ resolution because the detected sign image is converted into a log-polar image based on these two parameters: $\rho$ is a distance between sampling bins and the centroid and $\theta$ is a rotation angle of sampling bins in counterclockwise. Finally, our sign classifier can produce the same length of a column vector, $|\rho \times \theta| \times 1$ (e.g., a combination of the parameters, $\rho=32$ and $\theta=32$, resulting in $1024 \times 1$ ), for every sign.

TABLE I

THE NUMBER OF SIGN IMAGES FOR EACH TARGET CLASS.

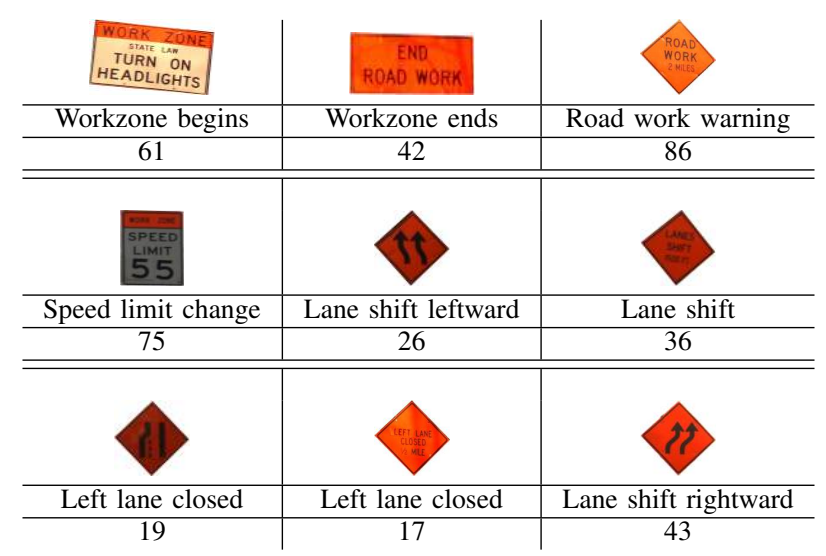

However the column vector still has a high-dimensionality space compared to the number of training data. We reduce the original dimension using principal component analysis (PCA). We then build an eigen-space from the training data and project a testing sign image in the log-polar coordinate space onto this eigen-space. 10 eigen bases cover more than $93 \%$ of the total variance of perfectly labeled input data. We use support vector machine (SVM) to classify the signs among those eigen bases.

\section{EXPERIMENTS}

This section details the experimentation measuring the performance of our sign workzone recognition system using images acquired under various weather conditions.

Our experiments measured the performance of detection and classification by executing two different detection types, 'detection only' and 'detection and tracking,' to show the improvement of our workzone recognition system when applying the tracking component. 'Detection only' executed our sign detector in every frame, while 'detection and tracking' executed our sign detector until it satisfied the predefined conditions. Then, our sign tracking was executed in the remaining frames until a sign disappeared. Our classifier predicted a target class of a sign once the sub-region image was generated by either the detection or tracking.

We collected video sequences at 640 by 480 resolution at $15 \mathrm{fps}$ under various environmental conditions. We labeled 5 video sequences among these with manually annotated ground truths of position and target class for the testing data. These testing data contained a sequence of a nominal highway, a workzone, and another nominal highway under various weather conditions. The top row of table II explains the total number of frames, the number of images workzone signs appearing on, and the weather condition.

To evaluate the performance of the localization, we used the metrics used for PASCAL object detection challenges [16]. An output bounding box, $o_{i}$, was considered a potential match to the ground truth bounding box, $g_{i}$, in a given image frame, $i$, if their area of overlap was greater than a predefined value, $\tau<\frac{\operatorname{Area}\left(o_{i} \cap g_{i}\right)}{\operatorname{Area}\left(o_{i} \cup g_{i}\right)}$. When a potential match was found in a given image, sign localization performance could be further analyzed by measuring the following: precision $=\frac{\operatorname{Area}\left(o_{i} \cap g_{i}\right)}{\operatorname{Area}\left(o_{i}\right)}$ and recall $=\frac{\operatorname{Area}\left(o_{i} \cap g_{i}\right)}{\operatorname{Area}\left(g_{i}\right)}$.

To clearly illustrate our experimental results, we detail one of them and summarize the whole performance. Fig. 3 illustrates one of the experimental results, i.e. video data $\mathrm{E}$, where the $x$-axis represents the number of image frames organized by time and the $y$-axis represents the workzone sign target class. When a classification output is matched with the ground truth labeling, we counted it as a correct classification. We further analyzed the classification result with respect to the performance of our localization. Fig. 3(b) magnifies the dashed rectangle in Fig. 3(a). "Road work warning" signs appeared 12 times before they disappeared from the camera's field of view. As shown, the recall of detection fluctuated while that of tracking was stable. We treated our localization method missed a sign when its 


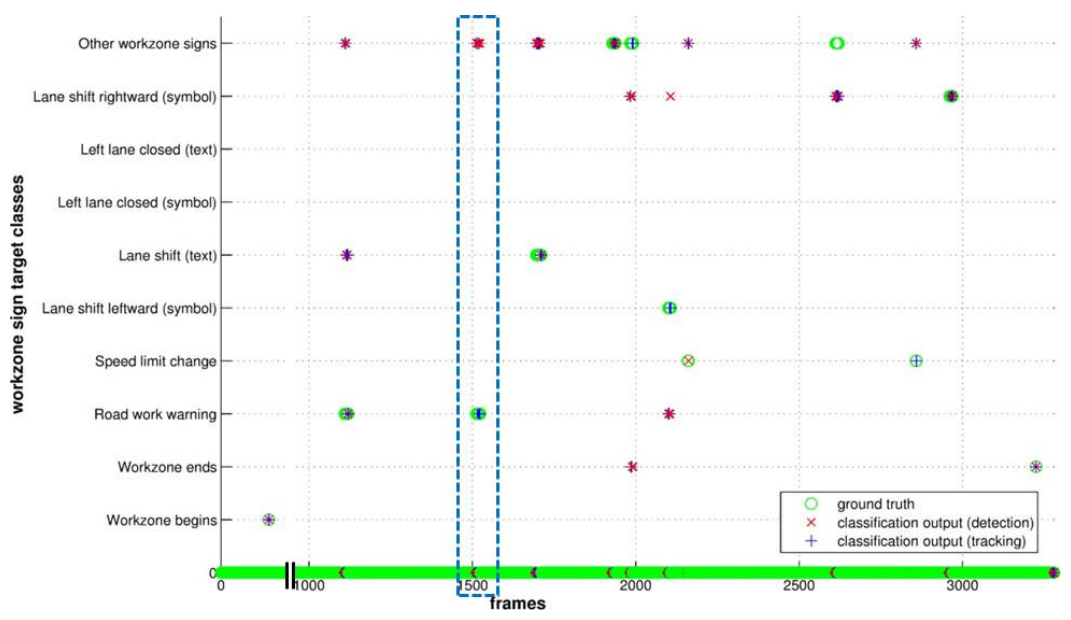

(a) A graph showing the summary of the test results.

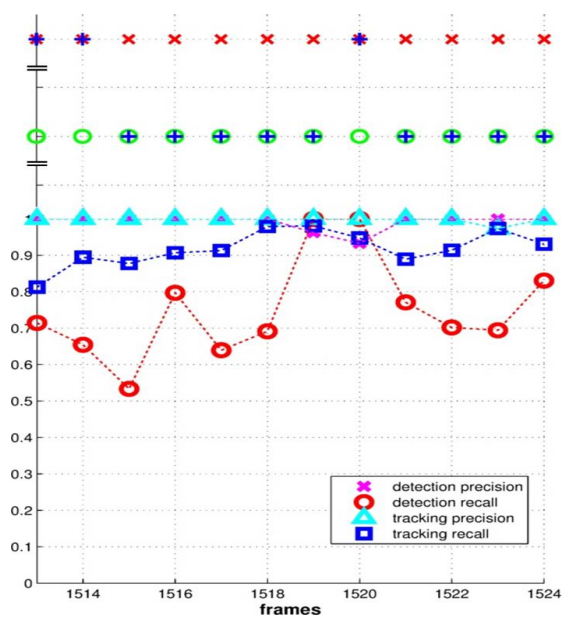

(b) This subfigure magnifies the dashed rectangle in Fig. 3 (a).
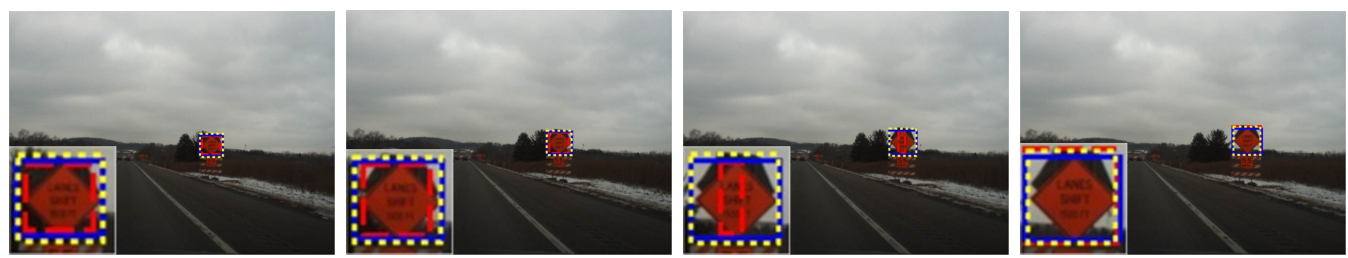

(c) Example images of localization results. The yellow dotted rectangles are ground truths, the blue solid rectangle are tracking results, and the red dashed rectnagle are detection results.

Fig. 3. Testing results of video data, E.

TABLE II

RESULTS OF OUR SYSTEM PERFORMANCE TESTS ON DIFFERENT DRIVING CONDITIONS.

\begin{tabular}{|c|c|c|c|c|c|c|}
\hline & & A & B & $\mathrm{C}$ & $\mathrm{D}$ & E \\
\hline Test video & $\begin{array}{c}\text { Number of images } \\
\text { Season } \\
\text { Weather }\end{array}$ & $\begin{array}{l}3305 / 445 \\
\text { Winter } \\
\text { Overcast }\end{array}$ & $\begin{array}{l}4232 / 646 \\
\text { Winter } \\
\text { Overcast }\end{array}$ & $\begin{array}{l}874 / 255 \\
\text { Spring } \\
\text { Sunny }\end{array}$ & $\begin{array}{l}3148 / 401 \\
\text { Spring } \\
\text { Sunny }\end{array}$ & $\begin{array}{l}3280 / 445 \\
\text { Spring } \\
\text { Rainy }\end{array}$ \\
\hline \multirow{2}{*}{ Detection } & $\begin{array}{l}\text { Number of tracked signs } \\
\text { Number of covered signs }\end{array}$ & $\begin{array}{c}97 \\
4\end{array}$ & $\begin{array}{c}117 \\
11\end{array}$ & $\begin{array}{c}49 \\
3\end{array}$ & $\begin{array}{c}152 \\
20\end{array}$ & $\begin{array}{l}98 \\
13\end{array}$ \\
\hline & Precision / Recall & $\begin{array}{l}\mathbf{0 . 9 9 9} / 0.879 \\
0.957 / \mathbf{0 . 9 0 5} \\
\end{array}$ & $\begin{array}{l}\mathbf{0 . 9 8 8} / \mathbf{0 . 8 7 0} \\
0.967 / 0.823 \\
\end{array}$ & $\begin{array}{l}\mathbf{0 . 9 8 9} / \mathbf{0 . 8 8 5} \\
0.967 / 0.874 \\
\end{array}$ & $\begin{array}{l}\mathbf{0 . 9 6 2} / 0.903 \\
0.854 / \mathbf{0 . 9 2 5} \\
\end{array}$ & $\begin{array}{l}\mathbf{0 . 9 8 3} / \mathbf{0 . 8 8 2} \\
0.956 / 0.832 \\
\end{array}$ \\
\hline \multirow{9}{*}{ Classification } & Workzone Begins & $\begin{array}{l}\mathrm{o} / 1.0 / 0.235 \\
\mathrm{o} / 1.0 / 0.235\end{array}$ & $\begin{array}{l}\mathrm{o} / \mathbf{0 . 6 6 7 / 1 . 0} \\
\mathrm{o} / 0.333 / 1.0\end{array}$ & $\begin{array}{l}\mathrm{o} / 0.125 / 1.0 \\
\mathrm{o} / \mathbf{0 . 2 5 0} / 1.0\end{array}$ & $\begin{array}{c}\mathrm{o} / 0.714 / 0.556 \\
\mathrm{o} / \mathbf{1 . 0} / \mathbf{1 . 0}\end{array}$ & $\begin{array}{l}\mathrm{o} / 1.0 / 1.0 \\
\mathrm{o} / 1.0 / 1.0\end{array}$ \\
\hline & Workzone Ends & $\begin{array}{l}\mathrm{o} / 1.0 / 1.0 \\
\mathrm{o} / 1.0 / 1.0\end{array}$ & $\begin{array}{l}\mathrm{o} / 1.0 / 1.0 \\
\mathrm{o} / 1.0 / 1.0\end{array}$ & $\begin{array}{l}\mathrm{o} / 1.0 / 1.0 \\
\mathrm{o} / 1.0 / 1.0\end{array}$ & $\begin{array}{l}\mathrm{o} / 1.0 / 1.0 \\
\mathrm{o} / 1.0 / 1.0\end{array}$ & $\begin{array}{c}\mathrm{o} / 1.0 / 0.2 \\
\mathrm{o} / 1.0 / 0.25\end{array}$ \\
\hline & Road work & $\begin{array}{l}\mathrm{o} / \mathbf{0 . 8 6 7 / 1 . 0} \\
\mathrm{o} / 0.533 / 1.0\end{array}$ & $\begin{array}{l}\mathrm{o} / \mathbf{0 . 5 8 5} / 0.774 \\
\mathrm{o} / 0.366 / 0.714 \\
\end{array}$ & $\begin{array}{c}\mathrm{o} / \mathbf{0 . 6 8 8} / 1.0 \\
\mathrm{o} / 0.0625 / 1.0\end{array}$ & $\begin{array}{c}\mathrm{o} / \mathbf{0 . 6 6 7 / 0 . 8 5 7} \\
\mathrm{o} / 0.5 / \mathbf{1 . 0} \\
\end{array}$ & $\begin{array}{l}\mathrm{o} / \mathbf{0 . 4 7 8 / 0 . 7 8 6} \\
\mathrm{o} / 0.0870 / 0.25 \\
\end{array}$ \\
\hline & Speed limit change & N/A & N/A & N/A & $\begin{array}{l}\mathrm{x} / 0.0 / 0.0 \\
\text { o/0.4/1.0 }\end{array}$ & $\begin{array}{l}\mathrm{o} / 0.2 / 1.0 \\
\mathrm{o} / 0.2 / 1.0\end{array}$ \\
\hline & Lane shift leftward symbol & $\begin{array}{l}\mathbf{0} / \mathbf{0 . 5} / \mathbf{1 . 0} \\
\mathrm{x} / 0.0 / 0.0\end{array}$ & $\begin{array}{c}\mathbf{0} / \mathbf{0 . 2 3 1} / \mathbf{1 . 0} \\
\mathrm{x} / 0.0 / 0.0 \\
\end{array}$ & N/A & N/A & $\begin{array}{c}\mathbf{0} / \mathbf{0 . 6 2 5} / \mathbf{1 . 0} \\
\mathrm{x} / 0.0 / 0.0\end{array}$ \\
\hline & Lane shift text & $\begin{array}{l}\mathrm{o} / \mathbf{0 . 6 7 7} / 1.0 \\
\mathrm{o} / 0.647 / 1.0\end{array}$ & $\begin{array}{l}\mathrm{o} / \mathbf{0 . 4 4 4 4 / 1 . 0} \\
\mathrm{o} / 0.222 / 1.0\end{array}$ & N/A & N/A & $\begin{array}{c}\mathrm{o} / \mathbf{0 . 2 5} / \mathbf{0 . 4} \\
\mathrm{o} / 0.125 / 0.333\end{array}$ \\
\hline & Left lane closed symbol & N/A & N/A & N/A & $\begin{array}{l}\mathrm{o} / 0.625 / 1.0 \\
\mathrm{o} / \mathbf{0 . 8 1 3} / 1.0\end{array}$ & N/A \\
\hline & Left lane closed text & N/A & N/A & N/A & $\begin{array}{l}\mathrm{o} / 0.143 / 1.0 \\
\mathrm{o} / 0.143 / 1.0\end{array}$ & N/A \\
\hline & Lane shift rightward symbol & N/A & $\begin{array}{l}\mathrm{x} / 0.0 / 0.0 \\
\mathrm{x} / 0.0 / 0.0\end{array}$ & N/A & $\begin{array}{c}\mathrm{o} / 1.0 / 0.7 \\
\mathrm{o} / 1.0 / 0.778\end{array}$ & $\begin{array}{l}\mathrm{o} / 1.0 / \mathbf{0 . 4 5} \\
\mathrm{o} / 1.0 / 0.356\end{array}$ \\
\hline
\end{tabular}

PASCAL measurement was less than 0.65. Our detector missed at frame 1515 and 1517. On the other hand, our tracking module didn't miss any signs during these frames and provided bounding boxes with high precision and recall, which led to a better classification performance.
We evaluated the performance of our workzone sign recognition system in terms of localization and classification. For the localization performance, we counted the number of signs that were missed by our sign detection module, but covered by our sign tracking module. Also, we calculated the 
averaged precision and recall of each instance. For the classification performance, we evaluated it in terms of nine target classes. For comparison, two different types of localization algorithms were executed: 'tracking' and 'detection only.' Table II further details the experimental results. The precision and recall values were calculated from only the frames which were executed on the tracking. (e.g., all the detection performance before the tracking started were ignored.) The first row in each cell represents the performance when tracking module is included while the second row represents the performance when only detection module is executed. In regard of the detection performance, our tracker successfully covered signs which were missed by our sign detector. For example, our sign tracking covered 13 missed signs from our sign detector. Furthermore, our tracker provided higher precision and recall than our detector which led to the better performance of the classification. Our recognition performance is improved with tracking except one test data, D. Our tracking system crops the potential sign region using the mode and standard deviation of the intensity within the bounding box. However, this sometimes over-crops the sign, which causes misclassification. Most of the degraded performance of the classification on D comes from this reason. Also, as explained in section III-A, we heuristically increase the bounding box for a rectangular workzone sign which includes two colors; "workzone begins" and "speed limit change" signs. Our tracking system only tries to track the orange color at the top and increases the bounding box heuristically, which is prone to error when increasing the bounding box. We demonstrate that our system including tracking component not only identifies the workzone bounds but also recognizes the details of temporary changes.

\section{CONCLUSIONS AND FUTURE WORK}

We presented a kernel-based traffic sign tracking to improve the performance of our highway workzone recognition system. The experimental results demonstrated that our improved workzone recognition system could provide the information of temporary changes in workzones. Our contributions in this paper include a successful application of kernel-based sign tracking method, resulting in picking up missed signs from our detector and improvement in our sign detection performance, increasing the performance of our sign classification component.

We showed promising results, but there is still a room for improvement. The shape information of signs can be used to further improve the performance of our sign tracker. In addition, our sign detector may miss signs in practice due to under- or over-exposure. In order to handle these issues, we would like to investigate a color constancy algorithm that estimates the illumination response function of our vision sensor. Furthermore, we would like to explore 3D scene geometry in order to effectively reduce the region of interest for better detection. Finally, we would like to continue testing under various road conditions and illuminations in order to evaluate the capability of our workzone recognition.

\section{ACKNOWLEDGMENT}

This work was previously funded by the General MotorsCarnegie Mellon University Autonomous Driving Collaborative Research Laboratory (AD-CRL).

\section{REFERENCES}

[1] Claus Bahlmann, Ying Zhu, Visvanathan Ramesh, Martin Pellkofer, and Thorsten Koehler, A system for traffic sign detection, tracking, and recognition using color, shape, motion information, In Proceedings of IEEE Symposiums on Intelligent Vehicles (IV-05), pp. 250-260, 2005.

[2] Simon Baker and Iain Matthews, Lucas-Kanade 20 years on: a unifying framework, International Journal of Computer Vision (IJCV04), 56(3): 221-255, 2004.

[3] Long Chen, Qingquan Li, Ming Li, and Qingzhou Mao, Traffic sign detection and recognition for intelligent vehicle, In Proceedings of IEEE Symposiums on Intelligent Vehicles (IV-11), pp. 908-913, 2011.

[4] Dorin Comaniciu, Visvanathan Ramesh, and Peter Meer, Kernelbased object tracking, IEEE Transactions on Pattern Recognition and Machine Intelligence (PAMI-03), 25(5): 564-577, 2003.

[5] Navneet Dalal and Bill Triggs, Histograms of oriented gradients for human detection, In Proceedings of IEEE Computer Vision and Pattern Recognition, pp. 886-893, 2005.

[6] Ernst Dickmanns, Dynamic Vision for Perception and Control of Motion, Springer, 2007.

[7] Marcin L. Eichner and Toby P. Breckon, Integrated speed limit detection and recognition from real-time video, In Proceedings of IEEE Symposiums on Intelligent Vehicles (IV-08), pp. 626-631, 2008.

[8] Chiung-Yao Fang, Sei-Wang Chen, and Chiou-Shann Fuh, Road-sign detection and tracking, IEEE Transactions on Vehicular Technology, 52(4): 1329-1341, 2003.

[9] Yoav Freund and Robert E. Schapire, Experiments with a new boosting algorithm, In Proceedings of International Conference on Machine Learning (ICML-96), pp. 148-156, 1996.

[10] Jerome FriedMan, Trevor Hastie, and Robert Tibshirani, Additive logistic regression: A statistical view of boosting, Annals of Statistics, 28(2): 337-407, 2000.

[11] Miguel Ángel García-Garrido, Miguel Ángel Sotelo, and Ernesto Martín-Gorostiza, Fast traffic sign detection and recognition under changing lighting conditions, In Proceedings of IEEE Intelligent Transportation Systems Conference (ITSC-06), pp. 811-816, 2006.

[12] Wei Liu, Xue Chen, Bobo Duan, Hui Dong, Pengyu Fu, Huai Yuan, and Hong Zhao, A system for road sign detection, recognition and tracking based on multi-cues hybrid, In Proceedings of IEEE Symposiums on Intelligent Vehicles (IV-09), pp. 562-567, 2009.

[13] Luis David Lopez and Olac Fuentes, Color-based road sign detection and tracking, In Proceedings of International Conference on Image Analysis and Recognition (ICIAR-07), pp. 1138-1147, 2007.

[14] Mirko Meuter, Anton Kummert and Stefan Müller-Schneiders, 3D traffic sign tracking using a particle filter, In Proceedings of IEEE Conference on Intelligent Transportation Systems (ITSC-08), pp. 168173, 2008.

[15] Montemerlo and et al., Junior: The stanford entry in the urban challenge. Journal of Field Robotics: Special Issues on the 2007 DARPA Urban Challenge, 25(9): 569597, 2008.

[16] Young-Woo Seo, David Wettergreen, and Wende Zhang, Recognizing temporary changes on highways for reliable autonomous driving, In Proceedings of IEEE International Conference on Systems, Man, and Cybernetics (SMC-12), pp. 3021-3026, 2012.

[17] George Siogkas and Evangelos Dermatas, Detection, tracking and classification of road signs in adverse conditions, In Proceedings of IEEE Mediterranean Electrotechnical Conference (MELECON-06), pp. 537-540, 2006.

[18] Chuck Thorpe, Todd Jochem, and Dean Pomerleau, The 1997 automated highway free agent demonstration, In Proceedings of International Symposium on Robotics Research, 1997.

[19] Chris Urmson and et al., Autonomous driving in urban environments: Boss and the urban challenge. Journal of Field Robotics: Special Issues on the 2007 DARPA Urban Challenge, 25(8): 425-466, 2008.

[20] U.S. Department of Transportation, Federal highway administration, Manual on uniform traffic control devices for streets and highways, http://mutcd.fhwa.dot.gov/, 2009 Edition. 